\title{
Especie nueva de Eritrachys (Hemiptera: Pentatomidae: Ochlerini) de Ecuador
}

\section{A new species of Eritrachys (Hemiptera: Pentatomidae: Ochlerini) from Ecuador}

\author{
Guillermina Ortega-León ${ }^{1 *}$ y Donald B. Thomas² \\ ${ }^{1}$ Instituto de Biología, Universidad Nacional Autónoma de México, Apartado postal 70-153, 04510 México, D.F., México \\ ${ }^{2}$ Kika de la Garza Subtropical Agriculture Research Center, United States Department of Agriculture ARS 2413 E. Hwy 83, Weslaco, 78596 TX, USA. \\ *Correspondencia: ortegag@ibiologia.unam.mx
}

\begin{abstract}
Resumen. Se describe e ilustra Eritrachys brailovskyi, una especie nueva del género Eritrachys Ruckes recolectada en Ecuador. Se describe e ilustra el macho de E. bituberculata Ruckes con nuevos registros para Panamá y Ecuador, y se da una clave para la separación de las especies del género.
\end{abstract}

Palabras clave: chinches hediondas, descripciones, Costa Rica, Panamá, Ecuador, bituberculata, brailovskyi.

\begin{abstract}
A new species of to the genus Eritrachys Ruckes, E. brailovskyi, collected in Ecuador, is described and illustrated. The male of E. bituberculata Ruckes is described and illustrated, with new records from Panama and Ecuador. A key to the species of the genus is provided.
\end{abstract}

Key words: stink bugs, descriptions, Costa Rica, Panama, Ecuador, bituberculata, brailovskyi.

\section{Introducción}

Ruckes (1959) describió el género Eritrachys con base en 2 ejemplares hembras; denominó E. bituberculata a la especie tipo, y colocó el género Eritrachys en la tribu Halyini, por el largo tubérculo antenal claramente visible dorsalmente, el surco abdominal para recibir el rostro y la depresión en la superficie dorsal del segmento terminal del tarso posterior. También lo relacionó con Hemingius Distant, separándolo de este último por los ojos pedunculados y alejados del margen anterior del pronoto, el largo del rostro, el margen pronotal anterior estrecho y el escutelo corto y con el ápice estrecho. Sin embargo, con fundamento en Rolston (1981, 1992), consideramos que debido a que la base del labium es caudal al límite anterior de los ojos, la posición de los tricobotrios lateral a la abertura espiracular sobre los esternitos VI y VII, y a la superficie dorsal excavada del III tarsómero tarsal de las patas posteriores, el género Eritrachys pertenece a la tribu Ochlerini, vinculándose principalmente con Ochlerus Spinola.

En este trabajo se describen e ilustran 2 especies: una especie nueva para Eritrachys proveniente del Ecuador, y el macho de E. bituberculata, ampliando su distribución a Panamá y Ecuador. Además de las características

Recibido: 23 abril 2009; aceptado: 29 julio 2009 antes mencionadas, ésta presenta un aspecto sedoso de la superficie dorsal, cabeza y pronoto rugoso; ápice del escutelo estrecho, y la presencia en mayor o menor grado de un par de tubérculos en el área central del pronoto y del escutelo.

\section{Material y métodos}

Las medidas se expresan en milímetros. Los dibujos se realizaron con la ayuda de una cámara clara. El material se encuentra depositado en la Pontificia Universidad Católica del Ecuador, Quito, Ecuador (PUCE); en el Instituto de Biodiversidad, Heredia, Costa Rica (INBIO); y en la Colección Nacional de Insectos de la Universidad Nacional Autónoma de México (CNIN).

\section{Descripción}

Eritrachys bituberculata Ruckes (Fig. 1a, c, e, g, h)

Macho. Coloración. De pardo amarillento oscuro a pardo oscuro con puntuaciones pardo oscuras cercanas entre sí; son de color pardo oscuro la cabeza, el tercio anterior del pronoto, las placas de la cápsula genital, región ventral y los tres pares de patas; ojos y segmentos antenales II y III pardo rojizo a oscuro; segmentos antenales IV y $\mathrm{V}$, coxas 
y tarsos pardo amarillento; corium de pardo amarillo a oscuro con una mancha discal presente en la centro del hemiélitro, membrana hemielitral pardo translúcida.

Morfología. Cabeza mucho más ancha a través de los ojos que larga medianamente; márgenes externos del tylus ampliamente sinuados y ligeramente reflejados, con el ápice redondeado; juga más cortos que el tylus y con su ápice agudamente redondeado pero no angulado; vértice y base del tylus poco elevado y vagamente rugoso transversalmente; puntuaciones cercanas entre sí; ojos pedunculados; distancia entre pronoto y ojos menor a la distancia entre los ocelos; antena con 5 segmentos, segmento subclavado y excediendo el ápice de la cabeza, segmento II el más corto, siendo el III el más largo; búcula con el ápice fuertemente angulado y los márgenes unidos detrás de la línea posterior del ojo; segmento rostral I casi alcanzando la procoxa, últimos 3 segmento casi el doble del segmento basal. Pronoto más de 2 veces más ancho a través de los ángulos humerales que largo, ligeramente elevado en vista lateral; disco pronotal transversalmente con las rugosidades bien marcadas e irregulares, así como con un par de tubérculos setígeros fuertemente prominentes y cónicos, centrales y a cada lado de la cicatriz; área cicatricial no punteada; puntuaciones entre la ruga prominentes y profundas; disco provisto de numerosas setas cortas y dirigidas hacia atrás; húmero escasamente dilatado, con los ángulos obtusos y poco expuestos, márgenes anterolaterales ligeramente carenados, elevados y ligeramente trisinuados, el margen de la carina abruptamente truncado justo después del húmero formando una pequeña emarginación; margen anterior suavemente excavado para recibir la cabeza, extendiéndose ligeramente lateral y oblicuamente formando un pequeño ángulo recto; margen posterior estrecho. Escutelo un poco más largo que ancho en la base, área central basal algunas veces dilatada con 2 tubérculos o elevaciones en la parte central del disco perfectamente definidas, el resto del escutelo más o menos aplanado con el ápice moderadamente redondeado, presentando una ligera muesca mesial y alcanzando el $\mathrm{V}$ tergito; puntuaciones ampliamente separadas, finas, apicalmente no distinguibles o contrastando con el color del disco pronotal; ángulo basal sin puntuaciones, sin callosidades, y con numerosas sedas distribuidas sobre la superficie; hemiélitro con pequeña mancha discal presente, no punteada; margen apical estrecho; ángulo apical recto, membrana excediendo el ápice del abdomen. Abdomen. Conexivo ligeramente expuesto; ángulo de cada segmento redondeado y el ángulo del VI ligeramente obtuso. Patas. Tibias profundamente surcadas.

Genitalia. Macho. Pigóforo semirredondeado, en vista ventral, con concavidad media en forma de V abierta; placa evidente semicuadrada y con ángulos externos del margen posterior casi rectos. Parámeros alargados con un lóbulo ampliamente redondeado en el tercio anterior del margen externo y con una serie de finas sedas; lóbulo apical formando una proyección alargada, delgada en forma de dedo, y con el margen interno ligeramente curvado (Fig. 1h). Hembra. Muy semejante al macho de mayor tamaño. Cápsula genital con el gonocoxito I más o menos ampliamente ovalado, un poco más largo que ancho, con el margen apical redondeado; paratergito IX ligeramente romboidal; paratergito VIII subparalelo con el margen interno contiguo, margen externo recto (Fig. 1e). Medidas. Longitud total $\widehat{\jmath}$ 9.6, +11.04 ; ancho de la cabeza

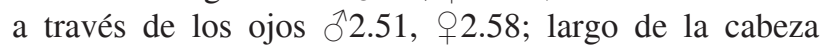

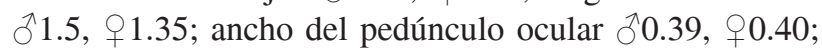
largo del pedúnculo ocular $\widehat{0} 0.27,90.21$; distancia entre

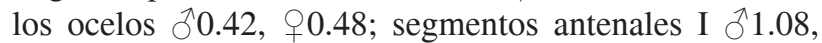

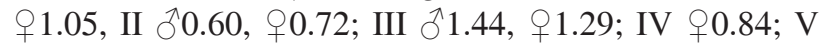

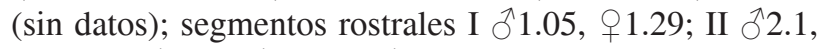

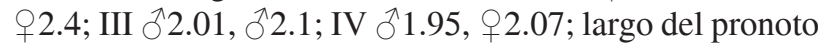

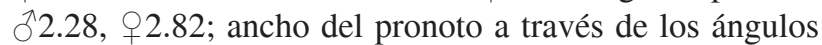

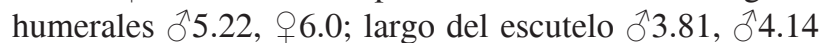
ancho del escutelo en la base $\precsim 3.42$, $\$ 3.64$.

\section{Resumen taxonómico}

Esta especie fue descrita por Ruckes con base en 2 hembras recolectadas en Costa Rica, Turrialba, mayo de 1944, col. Franz Schrader.

Nuevos registros. Costa Rica, 1 macho y 1 hembra: provincia Guanacaste, Estado Pitilla, 700 m, 9 km S Santa Cecilia, P. N. Guanacaste, 1.II.1992; col. C. Moraga. Macho depositado en INBIO y hembra en CNIN. Ecuador, 1 macho y 1 hembra: Pichincha Mundo $1800 \mathrm{~m}, 78^{\circ} 46^{\prime} \mathrm{O} 00^{\circ} 02^{\prime} \mathrm{S}$, 6.XI.1999, col. Francisco Prieto. Macho depositado en PUCE. Hembra depositada en CNIN. Ecuador, macho: Pichincha, Los Bancos III.1995, col. G. Onore. Ecuador, 1 hembra: Pichincha, Nenegal, 1400m, 0008'06' 'N 7842'00'’O, 16.IV.2000, col. F. Villamaría. Panamá, macho: provincia Campana, $2785 \mathrm{~m}, 08^{\circ} 40.920^{\prime} \mathrm{N}$, 7955.731'O; 28.V.2008; col. D.C. Robacker; ejemplares donados de la Colección D. B. Thomas, depositados en CNIN. Panamá, 1 macho y 1 hembra: Coclé, provincia El Valle, 7.I.1984, col. J. E. Wappes. Macho y hembra depositados en CNIN.

Eritrachys brailovskyi n. sp. (Fig. 1b, d, f, i, j)

Diagnosis. Jugum rebasando al tylus, uniéndose apicalmente, margen del jugum fuertemente reflejado; búcula ensanchada y cuadrada, concluyendo en un pequeño triángulo apical; pronoto fuertemente elevado, con un par 

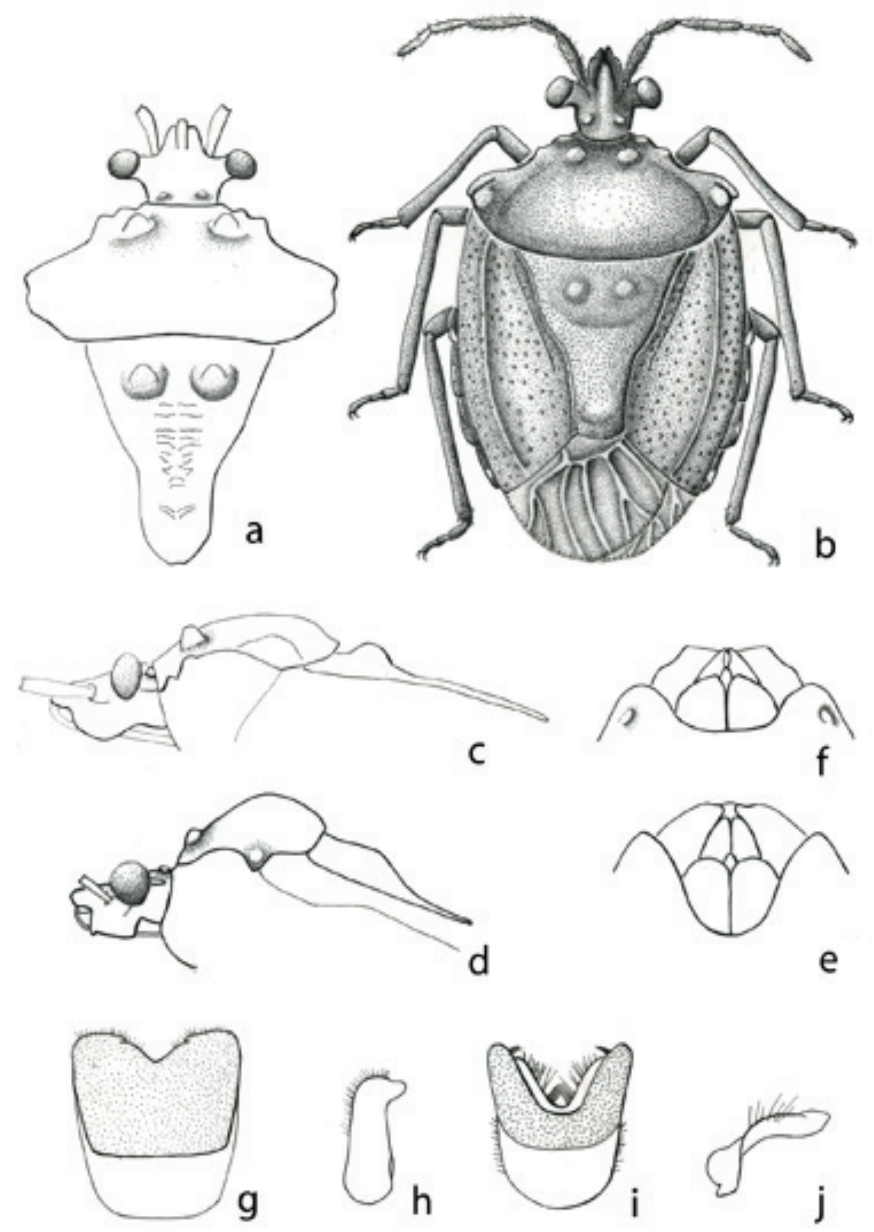

Figura 1. a-j. Eritrachys Ruckes. Vista dorsal: a), E. bituberculata; b), E. brailovskyi; c-d), vista lateral de la cabeza, pronoto y escutelo; c), E. bituberculata; d), E. brailovskyi; e-f), vista dorsal de las placas genitales: e), E. bituberculata; f), E. brailovskyi; g-i), vista dorsal del pigóforo: g), E. bituberculata; i), E. brailovskyi; h-j), vista lateral del parámero: h), E. bituberculata y j), E. brailovskyi.

de pequeños tubérculos setígeros; pigóforo en vista ventral con el margen anterior profundamente cóncavo, con la base recta; lóbulo apical del parámero semirredondeado, ligeramente angulado, nunca en forma de dedo, con el margen interno curvado formando una semiespiral.

Holotipo macho. Coloración general pardo rojizo a pardo oscuro, casi negro, con puntuaciones pardo oscuras esparcidas en todo el cuerpo, escasa pubescencia corta, amarillo oro; cabeza y patas pardo oscuro casi negro; ocelos de rojo a blanquecinos; de color pardo oscuro; ojos, segmentos antenales I, II, III, segmentos rostrales, pronoto, escutelo, corium, abdomen y espiráculos; IV segmento antenal y mitad apical del V pardo claro; mitad basal del V segmento amarillo; membrana hemielitral pardo translúcido; de amarillo ocre, una mancha media en el margen externo de cada segmento abdominal y base de los tricobotrios; de pardo rojizo a pardo amarillento la base de las coxas.

Morfología. Cabeza más ancha a través de los ojos que larga medialmente, márgenes laterales del jugum fuertemente reflejados, tylus más corto que los juga, los ápices de los juga se unen por delante del tylus; región media del tylus levemente elevada, ligeramente rugosa con o ninguna seda, las cuales si están presentes muy largas y espaciadas; ojos grandes y pedunculados; con el pedúnculo grueso y corto, segmento antenal I excediendo el ápice de la cabeza, el segmento II el más pequeño y el III el más largo; antena escasamente pubescente con excepción del segmento $\mathrm{V}$ que se encuentra cubierto con sedas pequeñas y finas; búcula ensanchada y cuadrada, margen anterior concluyendo en un pequeño triángulo apical, con el margen posterior terminando abruptamente en una línea recta; segmento rostral I no llega a las procoxas, rostro alcanzando el ápice del VII segmento abdominal. Pronoto fuertemente elevado, más de 2 veces más ancho a través de los ángulos humerales que largo; fuertemente rugoso, con un par de tubérculos setígeros levemente elevados; disco pronotal sin sedas; ángulos humerales hinchados con los ápices redondeados, margen lateroposterior carenado levemente elevado terminando en un pequeño diente; márgenes laterales con una concavidad mesial, márgenes antero-laterales levemente lobulados; márgenes posterolaterales elevados y angulados. Escutelo. Más largo que ancho; con 2 tubérculos cónicos levemente elevados en el área central basal, rugoso, con el ápice liso casi cuadrado, sin una muesca mesial, con puntuaciones separadas, sin callosidades, sin o con muy escasas setas pequeñas; conexivo ligeramente expuesto, ápice de cada segmento recto. Patas. Tibias profundamente surcadas.

Genitalia. Pigóforo más largo que ancho, lóbulos del margen anterior redondeados, margen medio profundamente cóncavo con la base recta fuertemente pubescente; placa ligeramente redondeada; parámeros con el lóbulo basal redondeado, margen interno curvado formando una semiespiral, con el margen apical ligeramente redondeado y angulado, nunca en forma de dedo, presentando setas largas en su porción media (Fig. 1j). Hembra semejante al macho, de mayor tamaño; gonocoxito I de la cápsula genital casi tan largo como ancho y con el margen apical angulado; paratergito IX sobrepasando levemente el gonocoxito I, con los márgenes internos cóncavos, nunca unidos; margen anterior del paratergito VIII recto; tercio medio del margen lateral levemente cóncavo (Fig. 1f)

Medidas. Longitud total $\widehat{\jmath} 10.8$, +11.4 ; anchura de la cabeza a través de los ojos $\bigcirc^{\top} 2.4, q 2.46$, largo de la cabeza

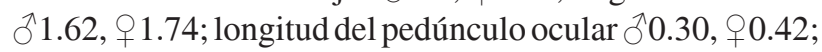


Clave para separar las especies de Eritrachys Ruckes

1.- $\quad$ 1. Tylus clara o ligeramente más largo que los juga, éstos nunca se unen apicalmente (Fig.1 a); pronoto no elevado; pronoto y escutelo con 2 tubérculos perfectamente definidos y elevados (Fig. 1c) lóbulo apical de los parámeros en forma de dedo (Fig. 1h). E. bituberculata Ruckes

1'.- $\quad 1$ '. Tylus más corto que los juga, éstos uniéndose apicalmente (Fig. 1b); pronoto fuertemente elevado; pronoto y escutelo con 2 tubérculos ligeramente marcados (Fig. 1d); lóbulo apical de los parámeros semiredondeado y ligeramente angulado (Fig. 1j).

E. brailovskyi n. sp.

ancho del pedúnculo ocular $\bigcirc 0.90,+0.84$; longitud entre los ojos y el pronoto 0.42 , 90.45 ; distancia entre los

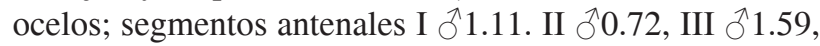
IV $\lesssim 0.90$, V $\lesssim 1.53$; segmentos rostrales I $\lesssim 1.26$, +1.35 ,

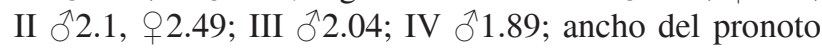
a través del humero $\widehat{\delta} 5.78$, 96.0 ; largo pronotal $\precsim 2.46$,

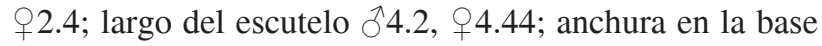
del escutelo ${ }^{\Im} 3.30$, 우‥42.

\section{Resumen taxonómico}

Material tipo. Holotipo macho, Ecuador, Cotopaxi, Las Pampas, 1800 m, 9.V.1997 col. G. Onore. Depositado en la Pontificia Universidad Católica del Ecuador, Quito, Ecuador (PUCE). Paratipo. Hembra, mismos datos. Depositado en la Colección Nacional de Insectos (CNIN) del Instituto de Biología, UNAM.

Etimología. Esta especie está dedicada al Dr. Harry Brailovsky Alperowitz, eminente hemipterólogo del Instituto de Biología, UNAM.

\section{Comentarios taxonómicos}

En E. brailovskyi los márgenes del jugum están fuertemente reflejados y unidos apicalmente, el tylus es mucho más corto que los juga (Fig. 1b), mientras que en $E$. bituberculata el tylus es más largo que los juga (Fig. 1a); el vértice y base del tylus rugoso y con pocas o ninguna seta, y cuando están presentes son largas y muy espaciadas, siendo en bituberculata cortas y densas; búcula ensanchada y cuadrada, con la porción basal concluyendo en un pequeño triángulo apical (Fig. 1d), en E. bituberculata de ligeramente recta a redondeada, desvaneciéndose posteriormente (Fig. 1c). El pronoto fuertemente elevado con un par de tubérculos levemente elevados (Fig. 1d), nunca llegando a ser como en E. bituberculata (Fig. 1c); escutelo con el ápice semicuadrado sin una muesca y con los tubérculos cónicos levemente elevados, nunca como en bituberculata; pigóforo en vista ventral con el margen profundamente cóncavo con la base recta (Fig. 1i), mientras que en bituberculata es en forma de $\mathrm{V}$ abierta (Fig. 1g); parámeros levemente helicoidales, con el lóbulo apical levemente angulado (Fig. 1j), nunca en forma de dedo como en E. bituberculata (Fig. 1h).

\section{Agradecimientos}

A Giovanni Onore, de la Pontificia Universidad Católica del Ecuador, Quito; a Jesús Ugalde, del Instituto Nacional de Biodiversidad de Heredia, Costa Rica; y a David A. Rider, de la North Dakota State University, por el préstamo del material aquí estudiado. A Luis Cervantes Peredo, del Instituto de Ecología, Xalapa, Veracruz, por la revisión y críticas al manuscrito. A Raúl Roldán Méndez y Viridiana Seng Iglesias, por su apoyo durante la toma de medidas. A los revisores anónimos por sus comentarios al texto.

\section{Literatura citada}

Rolston, H. L. 1981. Ochlerini a new tribe in Discocephalinae (Hemiptera: Pentatomidae). Journal New York Entomological Society 89:40-42.

Rolston, H. L. 1992. Key and diagnoses for the genera of Ochlerini (Hemiptera: Pentatomidae: Discocephalinae). Journal New York Entomological Society 100:1-41.

Ruckes, H. 1959. New genera and species of pentatomids from Panama and Costa Rica (Heteroptera: Pentatomidae). American Museum Novitates 1939:1-18. 